\title{
Drug-Drug Interaction Risk Assessment of Esaxerenone as a Perpetrator by In Vitro Studies and Static and Physiologically Based Pharmacokinetic Models ${ }^{\mathbb{}}$
}

\author{
Makiko Yamada, Tomoko Ishizuka, Shin-ichi Inoue, Veronika Rozehnal, Thomas Fischer, \\ and Daisuke Sugiyama
}

Drug Metabolism and Pharmacokinetics Research Laboratories, Daiichi Sankyo Co., Ltd., Tokyo, Japan (M.Y., T.I., S.I., D.S.) and Tissue and Cell Research Center Munich, Daiichi Sankyo Europe GmbH, Martinsried, Germany (V.R., T.F.)

Received February 14, 2020; accepted June 22, 2020

\section{ABSTRACT}

Esaxerenone (CS-3150) is a novel, oral, nonsteroidal, selective mineralocorticoid receptor blocker approved for the treatment of hypertension in Japan. Here, the drug-drug interaction (DDI) potential of esaxerenone was evaluated in vitro, and its impact in clinical practice was estimated. Esaxerenone exhibited time-dependent inhibition and induction of CYP3A. When the clinical impacts of esaxerenone on the inhibition and induction of CYP3A were estimated separately by using a mechanistic static model, the predicted area under the curve ratios (AUCRs) of midazolam, a typical CYP3A substrate, were 1.80 and 0.31 , respectively, suggesting that the DDI potential of esaxerenone cannot be neglected. Because it was suggested that DDls mainly occur in the intestine, predictions using concentration-time profiles in each segment of the gastrointestinal tract were performed with GastroPlus, a physiologically based pharmacokinetic (PBPK) modeling software. The predicted AUCR of midazolam was approximately 1.2 , which is close to that in a clinical study, despite the difficulty of predicting DDls for compounds with both inhibition and induction effects. When only inhibition or induction was incorporated into a model, the AUCR of midazolam changed depending on the dosing period and dose level of esaxerenone and the timing of midazolam administration. However, the AUCR calculated by incorporating both effects remained almost constant. This study shows the ability of PBPK models to simulate weak DDls via intestinal CYP3A and that esaxerenone has low DDI potential as a perpetrator because of the offset of inhibition and induction.

\section{SIGNIFICANCE STATEMENT}

Weak CYP3A inhibition and/or induction sometimes cause DDIs in the intestine but not the liver. Because strong inhibitors maximally inhibit intestinal CYP3A, the predictability of weak DDIs in the intestine should be evaluated further. Here, we simulate the DDIs of esaxerenone as a perpetrator by using physiologically based pharmacokinetic modeling focusing on the intestine and offset of inhibition and induction.
Introduction

Esaxerenone (CS-3150) is a novel, oral, nonsteroidal, selective mineralocorticoid receptor blocker that was approved for the treatment of hypertension in Japan in January 2019. It is currently under investigation for the treatment of diabetic nephropathy. Esaxerenone inhibits the binding of aldosterone to mineralocorticoid receptors with no agonistic or antagonistic effects on glucocorticoid, androgen, or progesterone receptors (Arai et al., 2015a) and exhibits potent and persistent antihypertensive and cardiorenal protective effects in rat models (Arai et al., 2015a,b, 2016). In clinical settings, esaxerenone

https://doi.org/10.1124/dmd.120.090928.

S This article has supplemental material available at dmd.aspetjournals.org. reduces blood pressure in patients with hypertension in a dosedependent manner (Ito et al., 2019a, 2020), and the effect persists for 52 weeks in patients with insufficient hypotensive effect by reninangiotensin system inhibitors and calcium channel blocker (Rakugi et al., 2019). In addition, the reduction of urinary albumin in patients with type 2 diabetes with microalbuminuria underscores the renal protective effects of esaxerenone (Ito et al., 2019b). Because of its high membrane permeability (Yamada et al., 2019), esaxerenone is rapidly absorbed after oral administration with high bioavailability (Kurata et al., 2019). Although its major elimination route is metabolism, esaxerenone is considered to have limited potential to be a victim of drug-drug interactions (DDIs) with enzyme inhibitors because it is metabolized via multiple routes, such as oxidation, hydrolysis, and glucuronidation (Yamada et al., 2019; Kirigaya et al., 2020a).

ABBREVIATIONS: AUCR, area under the curve ratio; $\mathrm{AUCR}_{\mathrm{g}}, \mathrm{AUCR}$ in the gut; $\mathrm{AUCR}_{\mathrm{h}}, \mathrm{AUCR}$ in the liver; $\mathrm{AUCR}$ ind, $A \cup C R$ incorporating induction; $A U C R_{\text {inh }}, A U C R$ incorporating inhibition; $A U_{C R}$ tot $A U C R$ incorporating all mechanisms available; BCRP, breast cancer resistance protein; $C_{\text {ent }}$, simulated concentrations in the enterocytes; $\mathrm{Cl}$, confidence interval; $\mathrm{CL}$, clearance; DDI, drug-drug interaction; $\mathrm{E}_{\max }$, maximum induction effect; FDA, Food and Drug Administration; $F_{g}$, intestinal availability; $f_{u, \text { inc }}$, in vitro unbound fraction; $f_{u, p}$, unbound fraction in plasma; FPE, first-pass effect; $I_{\text {gut }}$, intestinal luminal concentration of the interacting drug calculated as [dose] / [250 mL]; $\mathrm{K}_{12}$, rate constants for the distribution of the drug to the second compartment; $\mathrm{K}_{21}$, rate constants for the distribution of the drug from the second compartment; $\mathrm{K}_{\mathrm{l}}$, concentration of test substance yielding the inactivation rate constant at the half of $\mathrm{k}_{\text {inact }} ; \mathrm{k}_{\text {inact }}$, maximum inactivation rate constant; MATE, multidrug and toxin extrusion; OAT, organic anion transporter; OATP, organic anion transporter polypeptide; OCT, organic cation transporter; P450, cytochrome P450; PBPK, physiologically based pharmacokinetic; P-gp, p-glycoprotein; PK, pharmacokinetic; $R_{b}$, blood/plasma concentration ratio; TDI, time-dependent inhibition; UGT, uridine $5^{\prime}$-diphospho-glucuronosyltransferase; $V_{c}$, central compartment volume. 
It is important to evaluate DDIs in detail to ensure patient safety, especially for drugs for lifestyle-related diseases, which are often used concomitantly with other drugs. Regulatory authorities such as the US Food and Drug Administration (FDA), European Medicines Agency, and Japan Ministry of Labor and Welfare require in vitro studies to assess DDIs for new drug applications. When in vitro studies suggest a drug has inhibitory or inducing potential on metabolic enzymes and transporters, the drug's effect in clinical settings is usually first estimated using a static model. However, static models tend to overestimate DDIs because of the assumption that high concentrations remain constant (Vieira et al., 2014). Therefore, physiologically based pharmacokinetic (PBPK) models are used as a more realistic predictive approach for estimating DDIs (Jones et al., 2015; Guo et al., 2018; Shebley et al., 2018). Although it is reported that PBPK models can be used to determine the necessity of clinical DDI studies (Hsueh et al., 2018), fewer PBPK models have been submitted to the FDA to simulate DDIs with the drug under study as a perpetrator rather than as a victim (Supplemental Table by Grimstein et al., 2019). Moreover, only a few drugs have been approved without clinical studies investigating whether they are a perpetrator of DDIs based on the PBPK model (Shebley et al., 2018). For compounds showing both inhibition and induction potential in vitro, the DDI guidance recommends simulating in vivo inhibition and induction separately because concurrent prediction may lead to a false-negative prediction (Food and Drug Administration, 2020), and this approach leads to conservative judgment. In addition, when predicting weak DDIs via CYP3A, in vivo interaction is governed by the change of enzyme activity in the intestine rather than that in the liver because drug concentrations are usually higher in enterocytes than hepatocytes (Yamada et al., 2020). The predictive performance of PBPK models to simulate weak DDIs via CYP3A for orally administered drugs should be evaluated further because most previous studies used strong inhibitors, whereby intestinal CYP3A was completely inhibited. For example, a clinical DDI study of lenvatinib was required by a regulatory authority because of the uncertainty of intestinal DDI prediction by PBPK analysis (Shebley et al., 2018).

In this study, we evaluated the inhibitory potential of esaxerenone on cytochrome P450 isoforms, UGT1A1, UGT2B7, and several transporters, as well as its inducing activity on CYP1A2, CYP2B6, and CYP3A4 in vitro. When the DDI risk in clinical settings could not be neglected by comparing the in vitro results with $\mathrm{C}_{\max }$, it was assessed using a mechanistic static model and PBPK modeling software (GastroPlus version 9.7.0009; Simulations Plus, Lancaster, CA). Because pharmaceutical companies use PBPK models from the early discovery stage, when only animal and in vitro data are available (Jones et al., 2015), we compared the DDI predictability between models created with or without human PK data. By using the final GastroPlus model developed with all available data, the effects of different dosing periods and dose levels of esaxerenone and the timing of midazolam administration on DDI were also simulated. The effect of offset between inhibition and induction is also discussed.

\section{Material and Methods}

Materials. Esaxerenone [(S)-1-(2-hydroxyethyl)-4-methyl- $N$-[4-(methylsulfonyl) phenyl]-5-[2-(trifluoromethyl) phenyl]-1H-pyrrole-3-carboxamide] (Fig. 1) was synthesized at Daiichi Sankyo Co., Ltd. (Tokyo, Japan). $\left[{ }^{14} \mathrm{C}\right]$ Esaxerenone was supplied by Sekisui Medical (Ibaraki, Japan). Pooled human liver microsomes (50 donors, mixed sex, lot number: 0910398) were purchased from Sekisui Medical. Fresh human hepatocytes from six donors (age: 48-89, male and female) were obtained from Human Tissue and Cell Research Foundation (Regensburg, Germany). Phenacetin, coumarin, bupropion, paclitaxel, diclofenac, bufuralol, chlorzoxazone, testosterone, omeprazole, phenobarbital, rifampicin, $\beta$-estradiol, and zidovudine were purchased from Sigma-Aldrich (St. Louis, MO). Mephenytoin was purchased from Toronto Research Chemicals (Toronto, Canada). Midazolam was purchased from Wako Pure Chemical Industries (Tokyo, Japan). Other reagents were commercially available and of special reagent grade, highperformance liquid chromatography grade, liquid chromatography-mass spectrometry grade, or equivalent.

In Vitro DDI Studies. Esaxerenone's potential for reversible and timedependent inhibition (TDI) of CYP1A2, CYP2A6, CYP2B6, CYP2C8, CYP2C9, CYP2C19, CYP2D6, CYP2E1, and CYP3A4 was investigated using pooled human liver microsomes. Its potential for reversible inhibition of UGT1A1 and UGT2B7 was also investigated using pooled human liver microsomes because esaxerenone is a substrate of UGT isoforms (Yamada et al., 2019). UGT1A1 and UGT2B7 were selected because the Japanese regulatory agency recommends assessing the inhibitory effects on the two isoforms for the compound mainly metabolized by UGT, as a relatively large number of medical products are known to be substrates of the two isoforms (Pharmaceutical Evaluation Division, Pharmaceuticals Safety and Environmental Health Bureau, Ministry of Labor and Welfare, 2018). The following probe substrates were used: $30 \mu \mathrm{M}$ phenacetin for CYP1A2, $1 \mu \mathrm{M}$ coumarin for CYP2A6, $100 \mu \mathrm{M}$ bupropion for CYP2B6, $10 \mu \mathrm{M}$ paclitaxel for CYP2C8, $5 \mu \mathrm{M}$ diclofenac for CYP2C9, $20 \mu \mathrm{M}(S)$ mephenytoin for CYP2C19, $10 \mu \mathrm{M}$ bufuralol for CYP2D6, $40 \mu \mathrm{M}$ chlorzoxazone for CYP2E1, $5 \mu \mathrm{M}$ midazolam and $50 \mu \mathrm{M}$ testosterone for CYP3A4, $15 \mu \mathrm{M} \beta$-estradiol for UGT1A1, and $600 \mu \mathrm{M}$ zidovudine for UGT2B7. Probe substrate concentrations were chosen to be equal to or less than $\mathrm{K}_{\mathrm{m}}$ values based on the literature. However, when calculating the CYP3A inactivation parameters, concentrations higher than $\mathrm{K}_{\mathrm{m}}$ were intentionally used to exclude the effects of competitive inhibition (i.e., $25 \mu \mathrm{M}$ for midazolam and $250 \mu \mathrm{M}$ for testosterone). The concentrations of human liver microsomes were as follows: $0.2 \mathrm{mg} / \mathrm{ml}$ for reversible cytochrome $\mathrm{P} 450$ inhibition, $2 \mathrm{mg} / \mathrm{ml}$ for TDI, and $0.05 \mathrm{mg} / \mathrm{ml}$ for UGT inhibition. The $\mathrm{IC}_{50}$ was calculated if more than $50 \%$ inhibition was observed at the maximum esaxerenone concentration tested $(100 \mu \mathrm{M})$. For the compounds for which $\mathrm{IC}_{50}$ was calculated, $\mathrm{K}_{\mathrm{i}}$ values were determined by conducting an additional experiment. For $\mathrm{P} 450$ isoforms, the inhibitory potential of esaxerenone was evaluated with or without 30 minutes of preincubation with the NADPHgenerating system. If the $\mathrm{IC}_{50}$ with preincubation was smaller than that without preincubation, the maximum inactivation rate constant $\left(\mathrm{k}_{\text {inact }}\right)$ and the concentration yielding the apparent inactivation rate constant at half of $k_{\text {inact }}\left(\mathrm{K}_{\mathrm{I}}\right)$ were calculated by conducting an additional experiment. The incubations were performed in duplicate. The potential of esaxerenone $(0.1-10 \mu \mathrm{M}$ for CYP1A2 and $0.1-30 \mu \mathrm{M}$ for all others) to induce CYP1A2, CYP2B6, or CYP3A4 was investigated using primary cultures of fresh human hepatocytes (three donors for CYP1A2 and another three donors for both CYP2B6 and CYP3A4). Phenacetin $O$-deethylation, testosterone $6 \beta$-hydroxylation, and bupropion hydroxylation were used to specifically determine CYP1A2, CYP3A4, and CYP2B6 enzyme activities, respectively, and mRNA levels were quantified by quantitative realtime polymerase chain reaction. Omeprazole $(50 \mu \mathrm{M})$, phenobarbital (10-3000 $\mu \mathrm{M})$, and rifampicin $(0.1-30 \mu \mathrm{M})$ were used as reference articles for CYP1A2, CYP2B6, and CYP3A4, respectively. Incubations were performed in duplicate for CYP1A2 and in triplicate for CYP2B6 and CYP3A4. If concentration-dependent

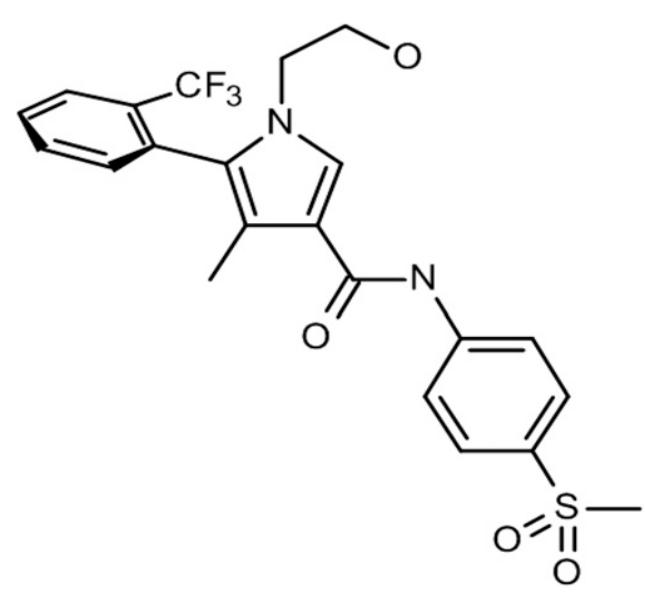

Fig. 1. Chemical structure of esaxerenone. 
induction was observed, the maximum induction effect $\left(\mathrm{E}_{\max }\right)$ and the $\mathrm{EC}_{50}$ were calculated. The inhibitory effects of esaxerenone on the transport of substrates by the following proteins were examined: human organic anion transporter (OAT) 1, OAT3, organic anion transporting polypeptide (OATP) 1B1, OATP1B3, organic cation transporter (OCT) 1, and OCT2 in transfected MDCK-II cells; human multidrug and toxin extrusion transporter (MATE) 1 and MATE2-K in transfected HEK293 cells; and human breast cancer resistance protein (BCRP) and p-glycoprotein (P-gp) in Caco-2 cells. The transfected cells were developed in-house. The following probe substrates were used: $10 \mu \mathrm{M}\left[{ }^{14} \mathrm{C}\right] \mathrm{metformin}$ for OCT1, OCT2, MATE1, and MATE2-K; $2 \mu \mathrm{M}\left[{ }^{3} \mathrm{H}\right] \mathrm{p}$-aminohippurate for OAT1; $0.75 \mu \mathrm{M}\left[{ }^{3} \mathrm{H}\right]$ estrone-3-sulfate for OAT3; $2 \mu \mathrm{M}\left[{ }^{3} \mathrm{H}\right]$ estradiol-17 $\beta$-D-glucuronide for OATP1B1; $10 \mu \mathrm{M}\left[{ }^{3} \mathrm{H}\right]$ cholecystokinin-8 for OATP1B3; $100 \mathrm{nM}\left[{ }^{3} \mathrm{H}\right]$ digoxin for P-gp; and $25 \mathrm{nM}$ $\left[{ }^{3} \mathrm{H}\right]$ genistein for BCRP. Incubations were performed in triplicate, and the incubation time was within the linear uptake range: 5 minutes for OCT1, OCT2, OAT1, OAT3, OATP1B1, and OATP1B3; 1 minute for MATE1; 2 minutes for MATE2-K; and 120 minutes for P-gp and BCRP. More details of the method are shown in the Supplemental Methods.

In Vitro Protein Binding to Human Plasma and Human Liver Microsomes. To determine protein binding to plasma, esaxerenone methanol solution (final concentration: $0.03,0.3$, or $3 \mu \mathrm{g} / \mathrm{ml}$ ) was added to the plasma of healthy male volunteers $(n=3)$. To determine protein binding to microsomes, $\left[{ }^{14} \mathrm{C}\right]$ esaxerenone ethanol solution (final concentration: $50 \mu \mathrm{M}$ ) was added to human liver microsomes ( 0.2 or $2 \mathrm{mg}$ protein $/ \mathrm{ml})$. The samples were incubated at $37^{\circ} \mathrm{C}$ for 10 minutes. Each sample was then transferred to ultracentrifugation tubes, a portion of the sample was collected from the tube as a total concentration sample, and each tube was ultracentrifuged at $436,000 \mathrm{~g}$ at $4^{\circ} \mathrm{C}$ for 140 minutes. After ultracentrifugation, a portion of the supernatant of each sample was collected as an unbound fraction sample. The concentrations of esaxerenone and $\left[{ }^{14} \mathrm{C}\right]$ esaxerenone were determined by liquid chromatography-tandem mass spectrometry and liquid scintillation counter, respectively. The unbound fraction was calculated by dividing the concentration in supernatant by that in plasma or microsomes. Plasma protein binding was calculated by subtracting the unbound fraction (\%) from 100.

In Vitro Blood/Plasma Concentration Ratio. To the blood of healthy male volunteers $(n=3),\left[{ }^{14} \mathrm{C}\right]$ esaxerenone methanol solution (final concentration: 0.03 , 0.3 , or $3 \mu \mathrm{g} / \mathrm{ml}$ ) was added, and the samples were incubated at $37^{\circ} \mathrm{C}$ for 10 minutes. A portion of each blood sample was collected into a vial. The remaining blood was centrifuged at $8000 \mathrm{~g}$ at $37^{\circ} \mathrm{C}$ for 5 minutes to separate plasma. A portion of each resultant plasma sample was collected into a vial. Radioactivity was measured by liquid scintillation counter. The blood/plasma concentration ratio $\left(\mathrm{R}_{\mathrm{b}}\right)$ was calculated by dividing the radioactivity of blood by that of plasma.

Static Model Analysis for DDI. The effects of esaxerenone on P-gp and BCRP were predicted by using a basic static model in accordance with the guidance for DDI by the FDA (Food and Drug Administration, 2020). The intestinal luminal concentration of the interacting drug $\left(\mathrm{I}_{\text {gut }}\right)$ was calculated as [dose $] /[250 \mathrm{ml}]=42.9 \mu \mathrm{M}$. If $\mathrm{I}_{\text {gut }} / \mathrm{IC}_{50}$ was less than 10 , the potential of esaxerenone to inhibit these transporters in vivo was considered low.

The effect of esaxerenone on the areas under the curve of midazolam for the inhibition and induction of CYP3A and CYP2B6 was evaluated by using a mechanistic static model with eq. 1 in accordance with the guidance for DDI by the FDA (Food and Drug Administration, 2020):

$$
\begin{aligned}
\text { AUCR }= & {\left[\frac{1}{\left[\left(\mathrm{~A}_{\mathrm{g}} \times \mathrm{B}_{\mathrm{g}} \times \mathrm{C}_{\mathrm{g}}\right) \times\left(1-\mathrm{F}_{\mathrm{g}}\right)+\mathrm{F}_{\mathrm{g}}\right]}\right] } \\
& \times\left[\frac{1}{\left[\left(\mathrm{~A}_{\mathrm{h}} \times \mathrm{B}_{\mathrm{h}} \times \mathrm{C}_{\mathrm{h}}\right) \times \mathrm{f}_{\mathrm{m}}+\left(1-\mathrm{f}_{\mathrm{m}}\right)\right]}\right]
\end{aligned}
$$

where $F_{g}$ is the fraction available after intestinal metabolism, and $f_{m}$ is the fraction of systemic clearance (CL) of a substrate mediated by the cytochrome P450 enzyme that is subject to inhibition/induction; subscripts " $h$ " and "g" denote the liver and gut, respectively. The $\mathrm{F}_{\mathrm{g}}$ of midazolam was calculated to be 0.54 based on its absolute bioavailability (0.30) and hepatic availability (0.56) (Thummel et al., 1996) and assuming the fraction absorbed $\left(\mathrm{F}_{\mathrm{a}}\right)$ was 1 . The $\mathrm{f}_{\mathrm{m}}$ of midazolam was set to 0.94 (Brown et al., 2005), and the $\mathrm{f}_{\mathrm{m}}$ of CYP2B6 substrate was set to 1 (maximum risk was evaluated). Terms A, B, and $\mathrm{C}$ represent reversible inhibition, TDI, and induction, respectively, and were calculated from eqs. $2-4$, respectively:

$$
\begin{gathered}
\mathrm{A}=\frac{1}{1+\frac{[\mathrm{I}]}{\mathrm{K}_{\mathrm{i}}}} \\
\mathrm{B}=\frac{\mathrm{k}_{\operatorname{deg}}}{\mathrm{k}_{\operatorname{deg}}+\frac{\mathrm{II}] \times \mathrm{k}_{\text {inact }}}{[\mathrm{I}]+\mathrm{K}_{\mathrm{I}}}} \\
\mathrm{C}=1+\frac{\mathrm{d} \cdot \mathrm{E}_{\max } \cdot[\mathrm{I}]}{[\mathrm{I}]+\mathrm{EC}_{50}}
\end{gathered}
$$

where $\mathrm{k}_{\mathrm{deg}}$ is the apparent first-order degradation rate constant of the affected enzyme ( 0.0005 minutes $^{-1}$ was used for both the liver and gut, respectively) (Zhang et al., 2009), and $d$ is the calibration factor (set to 1). The concentration of perpetrator in the intestine $\left(\left[[]_{\mathrm{g}}\right.\right.$ ) was calculated using eq. 5 (Rostami-Hodjegan and Tucker, 2004):

$$
[\mathrm{I}]_{\mathrm{g}}=\mathrm{F}_{\mathrm{a}} \times \mathrm{k}_{\mathrm{a}} \times \frac{\text { Dose }}{\mathrm{Q}_{\mathrm{en}}}
$$

where $\mathrm{k}_{\mathrm{a}}$ is the first-order absorption rate constant, and $\mathrm{Q}_{\mathrm{en}}$ is the blood flow through enterocytes $(18 \mathrm{~L} / \mathrm{h}$ ) (Yang et al., 2007a). For the concentration of the perpetrator in the liver $\left([\mathrm{I}]_{\mathrm{h}}\right)$, the maximum unbound plasma concentration at the inlet to the liver was calculated using eq. 6 (Ito et al., 1998):

$$
[\mathrm{I}]_{\mathrm{h}}=\mathrm{f}_{\mathrm{u}, \mathrm{p}} \times\left(\mathrm{C}_{\max }+\mathrm{F}_{\mathrm{a}} \times \mathrm{k}_{\mathrm{a}} \times \text { Dose } / \mathrm{Q}_{\mathrm{h}} / \mathrm{R}_{\mathrm{b}}\right)
$$

where $\mathrm{f}_{\mathrm{u}, \mathrm{p}}$ is the unbound fraction in plasma, $\mathrm{C}_{\max }$ is the maximal total (free and bound) inhibitor concentration in the plasma at steady state, and $\mathrm{Q}_{\mathrm{h}}$ is the hepatic blood flow (97 L/h per $70 \mathrm{~kg}$ ) (Yang et al., 2007b). The $\mathrm{k}_{\mathrm{a}}$ was calculated to be $1.19(1 / \mathrm{h})$ from the concentration-time profile of esaxerenone after $5 \mathrm{mg}$ oral administration (Kato et al., 2018) using Phoenix WinNonlin version 6.3 (Certara, Princeton, NJ). For $\mathrm{f}_{\mathrm{u}, \mathrm{p}}$ and $\mathrm{R}_{\mathrm{b}}$, the values obtained at esaxerenone $30 \mathrm{ng} / \mathrm{ml}$ in this study were used. $\mathrm{C}_{\max }$ after multiple oral administrations at $5 \mathrm{mg}$ was calculated as $87.2 \mathrm{ng} / \mathrm{ml}(\sim 0.2 \mu \mathrm{M})$ from the value after administration of $10 \mathrm{mg}$ for 10 days (Kato et al., 2018), assuming linear PK. $\mathrm{K}_{\mathrm{i}}, \mathrm{K}_{\mathrm{I}}$, and $\mathrm{EC}_{50}$ were corrected to the unbound value using the in vitro unbound fraction $\left(f_{u, \text { inc }}\right)$. The $f_{u \text {,inc }}$ for microsomes was obtained in this study; the $\mathrm{f}_{\mathrm{u}, \text { inc }}$ for hepatocytes was predicted to be 0.512 with Austin's equation (Austin et al., 2005) using GastroPlus. In addition to the AUCR incorporating all mechanisms available $\left(\mathrm{AUCR}_{\mathrm{tot}}\right)$, AUCRs incorporating inhibition and induction separately $\left(\mathrm{AUCR}_{\text {inh }}\right.$ and $A U C R_{\text {ind }}$, respectively) were calculated. The AUCRs based on the interactions in the gut and liver were also calculated separately $\left(\mathrm{AUCR}_{\mathrm{g}}\right.$ and $\mathrm{AUCR}_{\mathrm{h}}$, respectively).

Dynamic Model Analysis Using GastroPlus for DDI. GastroPlus version 9.7.0009 was used to construct the human PBPK model. Intestinal absorption and metabolism, systemic distribution and elimination, and DDI were simulated using the advanced compartmental absorption and transit model, compartmental PK model, and DDI module in GastroPlus, respectively. For parameters that are not specifically described below, the values incorporated into or predicted by GastroPlus were used (e.g., human physiologic parameters). The parameters used for the esaxerenone model are shown in Tables 1 and 2. The unbound fraction in enterocytes was set to $100 \%$, which was similar to our previous study (Yamada et al., 2020). This assumption was also used in the prediction of firstpass metabolism of CYP3A substrates in the intestine (Yang et al., 2007a). The midazolam model created in our previous study (Yamada et al., 2020) was used as a substrate model, and the parameters are shown in Supplemental Table 1. Three esaxerenone models were created with different human PK parameters to compare the DDI predictability. For model 1, human CL and the volume of distribution $\left(\mathrm{V}_{\mathrm{d}}\right)$ were predicted from monkey PK data after intravenous administration at $0.1 \mathrm{mg} / \mathrm{kg}$ (Yamada et al., 2017) with single-species allometric scaling using eqs. 7 and 8 (Lombardo et al., 2013a,b):

$$
\begin{aligned}
\mathrm{V}_{\mathrm{d}} \text { human } & =0.79 \times \mathrm{V}_{\mathrm{d}} \text { monkey } \\
\mathrm{CL} \text { human } & =0.40 \times \mathrm{CL} \text { monkey }
\end{aligned}
$$

To evaluate the maximum risk, bioavailability was assumed to be 1 . Because of the difficulty of predicting the distribution to the second compartment (i.e., the rate constants for the distribution of the drug to and from the second compartment; $\mathrm{K}_{12}$ and $\mathrm{K}_{21}$, respectively), a one-compartment model was used for model 1. For model 2, $\mathrm{CL}$, the central compartment volume $\left(\mathrm{V}_{\mathrm{c}}\right), \mathrm{K}_{12}$, and $\mathrm{K}_{21}$ were optimized to fit the concentration-time profiles after oral administration of esaxerenone $5 \mathrm{mg}$ (Kato et al., 2018). For model 3, CL, $\mathrm{V}_{\mathrm{c}}, \mathrm{K}_{12}$, and $\mathrm{K}_{21}$ were calculated using the 
data after intravenous administration of $5 \mathrm{mg}$ esaxerenone (Kurata et al., 2019), and first-pass effects (FPEs) were calculated and input into the model. The FPE in the liver was calculated from CL (assumed to be equal to hepatic clearance) by GastroPlus, and $\mathrm{F}_{\mathrm{g}}$ was calculated using the bioavailability of esaxerenone $(89.0 \%)$ (Kurata et al., 2019), assuming that the fraction absorbed was 1. Because the dissolution rate appeared too high for model 3, probably because of an inaccurate calculated dissolution rate from the observed average particle radius, the particle radius was increased from 3.5 to $10 \mu \mathrm{m}$ to fit the concentration-time profile after oral administration. DDI simulation was conducted using the dynamic simulation in the DDI module of GastroPlus. In addition to AUCR, $\mathrm{AUCR}_{\text {inh }}$ and $\mathrm{AUCR}_{\text {ind }}$ were calculated by separately incorporating inhibition parameters (reversible and TDI) and induction parameters, respectively. $\mathrm{K}_{\mathrm{i}}, \mathrm{K}_{\mathrm{I}}$, and $\mathrm{EC}_{50}$ corrected to the unbound values were similarly used for the static model analysis. For the $\mathrm{k}_{\mathrm{deg}}$ of CYP3A, 0.0005 minutes $^{-1}$ was used for both the gut and liver (Zhang et al., 2009). The ratio of intestinal availability was calculated as $\mathrm{AUCR}_{\mathrm{g}}$, and $\mathrm{AUCR}_{\mathrm{h}}$ was calculated by dividing the AUCR by $\mathrm{AUCR}_{\mathrm{g}}$. In the clinical study, esaxerenone $5 \mathrm{mg}$ was orally administered once daily for 14 days, and midazolam $2 \mathrm{mg}$ was orally administered simultaneously with esaxerenone on day 14 (Furuie et al., 2019). In the current study, to simulate the different durations of multiple doses of esaxerenone, the timing of midazolam dosing was set on days 1,2,3, and 14; to simulate the effect of dose staggering, the timing of midazolam dosing was set to $0,1,2$, and 12 hours after esaxerenone administration on day 14. In addition, DDI simulation was conducted with esaxerenone doses ranging from 1.25 to $10 \mathrm{mg}$. Model 3 was used for these simulations.

\section{Results}

\section{In Vitro DDI}

Inhibitory Potential of Esaxerenone on P450 and UGT Isoform Activities in Human Liver Microsomes. The $\mathrm{IC}_{50}$ values of esaxerenone on $\mathrm{P} 450$ isoforms with or without 30 minutes of preincubation are shown in Table 3. The Eadie-Hofstee plot and Dixon plot are shown in Supplemental Figs. 1 and 2, respectively. Inhibition was observed for CYP2B6 $\left(\mathrm{K}_{\mathrm{i}}=4.91 \mu \mathrm{M}\right)$, CYP2C8 $\left(\mathrm{K}_{\mathrm{i}}=13.1 \mu \mathrm{M}\right)$, CYP2C9 $\left(\mathrm{K}_{\mathrm{i}}=9.88\right.$ $\mu \mathrm{M}), \mathrm{CYP} 2 \mathrm{C} 19\left(\mathrm{~K}_{\mathrm{i}}=8.52 \mu \mathrm{M}\right)$, and CYP3A (midazolam; $\left.\mathrm{K}_{\mathrm{i}}=40.3 \mu \mathrm{M}\right)$.

TABLE 1

Input parameters of esaxerenone used in the GastroPlus simulation

\begin{tabular}{lc}
\hline Parameter (Unit) & \\
\hline Molecular weight & 466.47 \\
Solubility (mg/ml) & \\
Water [pH 8.5] $^{a}$ & 0.01 \\
FaSSIF $^{b}$ & 0.0395 \\
$\mathrm{FeSSIF}^{c}$ & 0.15 \\
$\operatorname{logD}[\mathrm{pH} 7.4]$ & $3.4^{a}$ \\
$\mathrm{p} K_{\mathrm{a}}$ & $10.7^{d}$ \\
$\mathrm{P}_{\text {eff }}\left(\mathrm{cm} / \mathrm{s} \times 10^{-4}\right)$ & $4.93^{e}$ \\
Particle radius $(\mu \mathrm{m})$ & $3.5^{a}$ \\
Models 1 and 2 & $10^{f}$ \\
Model 3 & $0.758^{g}$ \\
$\mathrm{R}_{\mathrm{b}}$ & $1.0^{\mathrm{g}}(0.867)^{h}$ \\
$\mathrm{f}_{\mathrm{u}, \mathrm{p}}(\%)$ & \\
$\mathrm{f}_{\mathrm{u} \text {,inc, microsomes }}$ & 0.434 \\
at $0.2 \mathrm{mg} / \mathrm{ml}$ & 0.066 \\
at 2 mg/ml & $0.512^{d}$ \\
$\mathrm{f}_{\mathrm{u}, \text { inc, hepatocytes }}$ & \\
\hline
\end{tabular}

$\log \mathrm{D}$, distribution coefficient; $\mathrm{P}_{\text {eff }}$, effective permeability.

${ }^{a}$ In-house data (data on file).

${ }^{b}$ Fasted-state simulated intestinal fluid ( $\mathrm{pH} 6.5$, bile salt concentration: $3 \mathrm{mM}$ ) predicted by GastroPlus.

${ }^{c}$ Fed-state simulated intestinal fluid $(\mathrm{pH} 5$, bile salt concentration: $15 \mathrm{mM}$ ) predicted by GastroPlus.

${ }^{d}$ Predicted by GastroPlus.

${ }^{e}$ Calculated using the Caco-2 permeability of esaxerenone (Yamada et al., 2019)

${ }^{f}$ Optimized value.

${ }^{g}$ At $30 \mathrm{ng} / \mathrm{ml}$ of esaxerenone.

${ }^{h}$ Adjusted by GastroPlus for possible binding to plasma lipids.
TABLE 2

PK parameters used for GastroPlus models

Model 1, PK parameters predicted from monkey data; model 2, PK parameters optimized using data after oral administration; model 3, PK parameters calculated using data after intravenous administration.

\begin{tabular}{lccl}
\hline & Model 1 & Model 2 & Model 3 \\
\hline CL $(1 / \mathrm{h}$ per kilogram $)$ & 0.0773 & 0.0631 & 0.0553 \\
$\mathrm{~V}_{\mathrm{c}}(1 / \mathrm{kg})$ & 1.06 & 1.27 & 0.332 \\
$\mathrm{~V}_{2}(1 / \mathrm{kg})$ & - & 0.520 & 0.896 \\
$\mathrm{~K}_{12}(1 / \mathrm{h})$ & - & 0.0111 & 1.26 \\
$\mathrm{~K}_{21}(1 / \mathrm{h})$ & - & 0.0272 & 0.467 \\
$\mathrm{FPE}_{\text {liver }}(\%)$ & - & - & 4.86 \\
$\mathrm{FPE}_{\text {gut }}(\%)$ & - & - & 6.41 \\
\hline
\end{tabular}

$\mathrm{V}_{2}$, second compartment volume.

The inhibition types were concluded as being the competitive type for all isoforms. Based on the $\mathrm{C}_{\max }$ after multiple oral administration of esaxerenone $5 \mathrm{mg}(\sim 0.2 \mu \mathrm{M})$, the possibility of clinically relevant PK changes of substrates via reversible inhibition of these enzymes was considered low. When testosterone was used as a CYP3A4 substrate, the $\mathrm{IC}_{50}$ exceeded $100 \mu \mathrm{M}$. Esaxerenone did not inhibit the specific activities of CYP1A2, CYP2A6, CYP2D6, or CYP2E1. However, esaxerenone exhibited a time-dependent inhibitory effect on the specific activity of CYP3A4. The $\mathrm{IC}_{50}$ of esaxerenone on the specific activities of CYP3A4 with 30 minutes of preincubation were smaller than those without preincubation (Table 3). Therefore, an inactivation study was conducted using midazolam and testosterone as CYP3A4 substrates. The calculated $\mathrm{k}_{\text {inact }}$ and $\mathrm{K}_{\mathrm{I}}$ of esaxerenone were 0.0235 minutes $^{-1}$ and $44.8 \mu \mathrm{M}$ for midazolam, respectively; those for testosterone were 0.0306 minutes $^{-1}$ and $54.6 \mu \mathrm{M}$, respectively (Table 3 ). The kinetic plot is shown in Supplemental Fig. 3.

The potential of esaxerenone to reversibly inhibit UGT1A1 and UGT2B7 was investigated using pooled human liver microsomes. The $\mathrm{IC}_{50}$ values (95\% confidence interval [CI]) for UGT1A1 and UGT2B7 were $23.6 \mu \mathrm{M}(15.6-31.6)$ and $>100 \mu \mathrm{M}$, respectively, and the $\mathrm{K}_{\mathrm{i}}$ (95\% CI) of esaxerenone was $12.4 \mu \mathrm{M}(10.6-14.1)$ in UGT1A1.

Induction Potential on P450 Isoform Activities in Fresh Human Hepatocytes. The potential of esaxerenone to induce CYP1A2, CYP2B6, or CYP3A4 was investigated using primary cultures of fresh human hepatocytes from three donors. Esaxerenone did not exhibit any cytotoxic effect. The mRNA fold induction and enzyme activity at the maximum concentration of each tested compound with omeprazole, rifampicin, or phenobarbital, which showed sufficient induction, as a reference are shown in Fig. 2. Esaxerenone did not induce CYP1A2 mRNA or activity but did exhibit induction potential for CYP2B6 and CYP3A4. Of note, the esaxerenone-induced increase in CYP3A4 activity was smaller than the increase in mRNA expression, probably because of the inhibitory potential of esaxerenone. Given that mRNA is generally more indicative of induction potential than metabolic activity (Food and Drug Administration, 2020), the $\mathrm{E}_{\max }$ and $\mathrm{EC}_{50}$ for CYP3A4 induction were calculated using mRNA data. However, for CYP2B6, the induction parameters were calculated using the metabolic activity because the fold induction of metabolic activity of CYP2B6 was greater than that of mRNA. The calculated values of esaxerenone and reference compounds (i.e., rifampicin for CYP3A4 and phenobarbital for CYP2B6) are shown in Table 4. The kinetic plot is shown in Supplemental Fig. 4. The $\mathrm{E}_{\max }$ and $\mathrm{EC}_{50}$ of esaxerenone for CYP3A4 were 24.0- to 61.2-fold and 4.1-16.4 $\mu \mathrm{M}$, respectively; those for CYP2B6 were 5.52- to 15.5-fold and 4.70-21.0 $\mu \mathrm{M}$, respectively.

Inhibitory Potential on Transporters. The inhibitory effects of esaxerenone on substrate transport by the following proteins were examined: OAT1, OAT3, OATP1B1, OATP1B3, OCT1, and OCT2 
TABLE 3

Inhibitory effects of esaxerenone on cytochrome $\mathrm{P} 450$ isoforms

Values in parentheses represent $95 \% \mathrm{CI}$.

\begin{tabular}{|c|c|c|c|c|c|}
\hline \multirow{3}{*}{$\begin{array}{l}\text { Cytochrome } \\
\text { P450 Isoform }\end{array}$} & \multicolumn{2}{|c|}{$\mathrm{IC}_{50}(\mu \mathrm{M})$} & \multirow{3}{*}{$\mathrm{K}_{\mathrm{i}}(\mu \mathrm{M})$} & \multirow{3}{*}{$\mathrm{k}_{\text {inact }}\left(\min ^{-1}\right)$} & \multirow{3}{*}{$\mathrm{K}_{\mathrm{I}}(\mu \mathrm{M})$} \\
\hline & \multicolumn{2}{|c|}{ Preincubation } & & & \\
\hline & $0 \mathrm{~min}$ & $30 \mathrm{~min}$ & & & \\
\hline CYP1A2 & $>100$ & $>100$ & NT & NT & NT \\
\hline CYP2A6 & $>100$ & $>100$ & NT & NT & NT \\
\hline CYP2B6 & $18.5(17.3-19.7)$ & $30.4(24.0-36.7)$ & $4.91(3.55-6.28)$ & NT & NT \\
\hline CYP2C8 & $49.6(43.1-56.0)$ & $53.7(48.0-59.3)$ & $13.1(7.51-18.8)$ & NT & NT \\
\hline CYP2C9 & $20.0(17.5-22.4)$ & $25.5(21.7-29.2)$ & $9.88(8.81-11.0)$ & NT & NT \\
\hline CYP2C19 & $31.4(22.9-39.9)$ & $33.7(24.4-43.0)$ & $8.52(4.36-12.7)$ & NT & NT \\
\hline CYP2D6 & $>100$ & $>100$ & NT & NT & NT \\
\hline CYP2E1 & $>100$ & $>100$ & NT & NT & NT \\
\hline CYP3A4 & $55.1(48.8-61.4)$ & $14.0(11.9-16.2)$ & $40.3(30.3-50.2)$ & $0.0235(0.0210-0.0261)$ & $44.8(31.5-58.1)$ \\
\hline CYP3A4 & $>100$ & $37.6(27.1-48.2)$ & NT & $0.0306(0.0290-0.0322)$ & $54.6(47.2-62.0)$ \\
\hline
\end{tabular}

NT, not tested.

in transfected MDCK-II cells; MATE1 and MATE2-K in transfected HEK293 cells; and human BCRP and P-gp in Caco- 2 cells. The $\mathrm{IC}_{50}$ (95\% CI) values of esaxerenone were as follows: $9.84 \mu \mathrm{M}(3.79-15.9)$ for OCT1, 31.0 $\mu \mathrm{M}(24.2-37.7)$ for OCT2, $9.70 \mu \mathrm{M}(6.78-12.6)$ for MATE1, 5.64 $\mu \mathrm{M}$ (4.38-6.91) for MATE2-K, $24.6 \mu \mathrm{M}$ (4.81-44.5) for BCRP, and $16.3 \mu \mathrm{M}(12.2-20.5)$ for P-gp. The kinetic plot is shown in Supplemental Fig. 5. The $\mathrm{IC}_{50}$ values of esaxerenone for OAT1, OAT3, OATP1B1, and OATP1B3 all exceeded $30 \mu \mathrm{M}$. Thus, considering systemic exposure, clinically relevant PK changes in substrates of OCT1, OCT2, MATE1, and MATE2-K are unlikely. Regarding P-gp and $\mathrm{BCRP}$, as $\mathrm{I}_{\text {gut }} / \mathrm{IC}_{50}$ were 2.63 and 1.74 , respectively, the potential of an in vivo DDI is considered to be low (i.e., an $\mathrm{I}_{\text {gut }} / \mathrm{IC}_{50}$ ratio $\geq 10$ indicates DDI potential).

\section{In Vitro Protein Binding and Blood/Plasma Concentration Ratio}

Protein binding to plasma and liver microsomes was determined using an ultracentrifugation method. The mean \pm S.D. plasma protein binding of esaxerenone 30,300 , and $3000 \mathrm{ng} / \mathrm{ml}$ was $99.0 \% \pm 0.3 \%$,

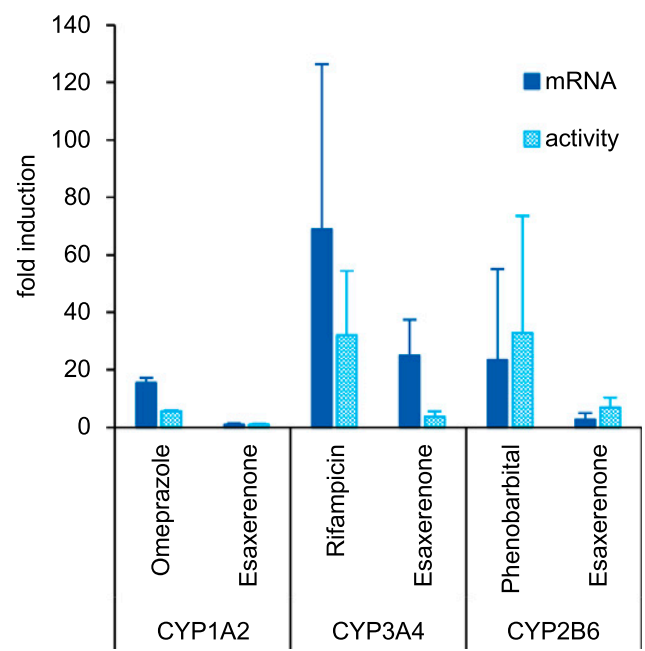

Fig. 2. Fold induction of mRNA and enzyme activities of CYP1A2, CYP3A4, and CYP2B6. Fold induction of mRNA and enzyme activities of CYP1A2, CYP3A4, and CYP2B6 were examined in primary cultured human hepatocytes, and the results at the maximum concentration of each compound tested are shown: omeprazole (50 $\mu \mathrm{M})$, rifampicin $(30 \mu \mathrm{M})$, phenobarbital $(3000 \mu \mathrm{M})$, and esaxerenone (10 $\mu \mathrm{M}$ for CYP1A2 and $30 \mu \mathrm{M}$ for CYP3A4 and CYP2B6). Data are expressed as means + S.D. of hepatocytes from three donors.
$98.5 \% \pm 0.5 \%$, and $98.2 \% \pm 0.3 \%$, respectively. The unbound fractions of esaxerenone $(50 \mu \mathrm{M})$ to liver microsomes $(0.2$ or $2 \mathrm{mg}$ protein $/ \mathrm{ml})$ were 0.434 and 0.066 , respectively. The mean \pm S.D. $R_{b}$ of esaxerenone 30,300 , and $3000 \mathrm{ng} / \mathrm{ml}$ was $0.758 \pm 0.114,0.772 \pm 0.067$, and 0.770 \pm 0.044 , respectively.

\section{DDI Risk Assessment Using a Mechanistic Static Model}

Among the isoforms tested, the smallest $\mathrm{K}_{\mathrm{i}}$ value was observed for CYP2B6, and TDI was observed for CYP3A. In addition, induction was observed for CYP2B6 and CYP3A4. To evaluate the necessity of clinical DDI studies of esaxerenone with CYP2B6 or CYP3A4 substrate, we analyzed DDI potential using a mechanistic static model. The predicted enzyme activity changes of CYP2B6 by reversible inhibition and induction were $1.00\left(\mathrm{~A}_{h}\right)$ and $1.02\left(\mathrm{C}_{\mathrm{h}}\right)$, respectively, indicating that the potential of a DDI via CYP2B6 is negligible in clinical settings. As for CYP3A4, the possibility of an intestinal DDI must be considered because of its high expression in enterocytes. The predicted fold changes in enzyme activity of CYP3A4 by reversible inhibition, TDI, and induction were $0.961\left(\mathrm{~A}_{\mathrm{g}}\right), 0.099\left(\mathrm{~B}_{\mathrm{g}}\right)$, and 5.76 $\left(\mathrm{C}_{\mathrm{g}}\right)$ in the gut and $1.00\left(\mathrm{~A}_{\mathrm{h}}\right), 0.946\left(\mathrm{~B}_{\mathrm{h}}\right), 1.03\left(\mathrm{C}_{\mathrm{h}}\right)$ times in the liver, respectively. These results indicate that TDI and induction in the intestine are important factors of a DDI with esaxerenone as a perpetrator. When inhibition and induction were considered separately, as recommended by the DDI guidance (Food and Drug Administration, 2020), the calculated $\mathrm{AUCR}_{\mathrm{inh}}$ and $\mathrm{AUCR}_{\mathrm{ind}}$ of midazolam were 1.80 and 0.31 , respectively. This suggests that DDI potential of esaxerenone cannot be neglected despite not being very strong. The AUCR calculated with both inhibition and induction was 1.30 .

\section{Dynamic Model Simulation of DDI via CYP3A Using GastroPlus}

GastroPlus models were created with three sets of human PK parameters: 1) predicted from monkey PK data by single-species allometric scaling, 2) optimized to fit clinical PK data after oral administration, and 3) calculated from clinical PK data after intravenous and oral administration. The PK parameters used for the simulation are summarized in Table 2. The simulated concentrations of esaxerenone in plasma and enterocytes (jejunum compartment 1 as an example) are shown in Fig. 3. Despite the different simulated plasma concentrations between models 1 and 2 (Fig. 3, A and B), the simulated concentrations in the enterocytes $\left(\mathrm{C}_{\text {ent }}\right)$ (Fig. 3, C and D) were the same and, hence, so were the simulated AUCRs because predicted $\mathrm{C}_{\mathrm{ent}}$ is mainly determined on the basis of solubility, dissolution rate, and membrane permeation. For model 3, FPE was calculated using absolute bioavailability (89.0\%) 
TABLE 4

Induction parameters of esaxerenone and reference compounds on CYP2B6 and CYP3A4

Primary cultures of fresh human hepatocytes from three donors were used. Parameters were calculated using the fold induction of metabolic activity for CYP2B6 and mRNA for CYP3A4. Parameters for CYP1A2 were not calculated, because the induction potential of esaxerenone was not observed.

\begin{tabular}{|c|c|c|c|c|c|c|}
\hline & & & No. 1 & No. 2 & No. 3 & Mean \pm S.D. \\
\hline \multirow[t]{4}{*}{ CYP2B6 } & \multirow{2}{*}{ Esaxerenone } & $\mathrm{E}_{\max }$ & 15.5 & 5.52 & 6.57 & $9.21 \pm 5.51$ \\
\hline & & $\mathrm{EC}_{50}(\mu \mathrm{M})$ & 4.70 & 7.64 & 21.0 & $11.1 \pm 8.7$ \\
\hline & \multirow[t]{2}{*}{ Phenobarbital } & $\mathrm{E}_{\max }$ & 215 & 8.98 & 51.4 & $91.8 \pm 108.8$ \\
\hline & & $\mathrm{EC}_{50}(\mu \mathrm{M})$ & 5170 & 968 & 12,700 & $6280 \pm 5940$ \\
\hline \multirow[t]{4}{*}{ CYP3A4 } & \multirow[t]{2}{*}{ Esaxerenone } & $\mathrm{E}_{\max }$ & 61.2 & 26.9 & 24.0 & $37.4 \pm 20.7$ \\
\hline & & $\mathrm{EC}_{50}(\mu \mathrm{M})$ & 16.4 & 4.10 & 10.9 & $10.5 \pm 6.1$ \\
\hline & \multirow{2}{*}{ Rifampicin } & $\mathrm{E}_{\max }$ & 171.5 & 54.3 & 18.9 & $81.6 \pm 79.9$ \\
\hline & & $\mathrm{EC}_{50}(\mu \mathrm{M})$ & 8.57 & 0.566 & 0.709 & $3.28 \pm 4.58$ \\
\hline
\end{tabular}

(Kurata et al., 2019) and input (4.86\% for liver and $6.41 \%$ for intestine). Because the dissolution rate predicted by particle radius appeared to be too high to capture absorption profile (i.e., time to reach maximum plasma concentration was too short), it was adjusted by optimizing particle radius, resulting in lower $\mathrm{C}_{\mathrm{ent}}$. The AUCRs in the gut and liver under conditions of inhibition only, induction only, and both inhibition and induction (i.e., total) calculated by models 1-3 using the same dosing condition as that in the clinical study are shown in Table 5. The calculated AUCRs of midazolam were similar among the three models, although $\mathrm{AUCR}_{\text {inh }}$ and $\mathrm{AUCR}_{\text {ind }}$ were slightly smaller in model 3 than those in model 1 or 2 . The AUCR $_{\text {tot }}$ calculated using the three models ranged from 1.17 to 1.23 , implying that esaxerenone has low DDI potential via both CYP3A inhibition and induction; moreover, these values are close to that reported in a clinical study $(\sim 1.2)$ (Furuie et al., 2019). The $\mathrm{AUCR}_{\mathrm{h}}$ calculated using all three models was approximately 1 , even when only inhibition or induction was incorporated. This suggests interactions would mainly occur in the intestine, whereas hepatic interaction is negligible given the weak interaction potency and low clinical exposure of esaxerenone as a perpetrator. The AUCRs calculated with respect to various scheduling of esaxerenone doses (5 mg once daily for $1,2,3$, or 14 days), timing of midazolam dose (i.e., $0,1,2$, or 12 hours after esaxerenone administration), or esaxerenone dose (i.e., 1.25, 2.5, 5, or $10 \mathrm{mg}$ ) are shown in Fig. 4. The $\mathrm{AUCR}_{\mathrm{inh}}$ of midazolam with single administration of esaxerenone $5 \mathrm{mg}$ was smaller than that with 14-day administration. The $\mathrm{AUCR}_{\mathrm{inh}}$ with 3-day administration was slightly smaller than that with 14-day administration. However, because induction was slightly weaker than that with 14-day administration, the $\mathrm{AUCR}_{\text {tot }}$ at days 3 and 14 was almost the same. Compared with simultaneous administration of midazolam and esaxerenone, administration of midazolam 1 or 2 hours after esaxerenone resulted in a larger $\mathrm{AUCR}_{\mathrm{inh}}$ and smaller $\mathrm{AUCR}_{\text {ind }}$ but a similar AUCR $_{\text {tot }}$. When midazolam was administered 12 hours after esaxerenone, the $\mathrm{AUCR}_{\text {inh }}$ was smaller than those with administration 1 or 2 hours after esaxerenone, probably because of enzyme turnover. When DDI simulation was conducted with various doses of esaxerenone (i.e., $1.25-10 \mathrm{mg}$ ), $\mathrm{AUCR}_{\text {inh }}$ increased and $\mathrm{AUCR}_{\text {ind }}$ decreased in a dose-dependent manner. However, AUCR $_{\text {tot }}$ remained almost constant owing to offset, i.e., the decrease in metabolic activity due to inhibition was alleviated by the increase in enzymes due to induction. Compared with the results of the static model, PBPK modeling simulated inhibition and induction more weakly (i.e., smaller $\mathrm{AUCR}_{\mathrm{inh}}$ and larger $\mathrm{AUCR}_{\mathrm{ind}}$, respectively) regardless of the conditions used (Fig. 4).

\section{Discussion}

This study assessed the DDI risks of esaxerenone as a perpetrator by evaluating DDIs in vitro as well as in static and PBPK models. The utility of the PBPK modeling software, GastroPlus, for the prediction of weak DDIs in the intestine is also discussed. Esaxerenone showed low or no risk of clinically relevant DDIs occurring via the inhibition of major enzymes or transporters, except for CYP3A, according to the static model analysis. Although the present results suggest low DDI potential via P-gp, we conducted a clinical study involving healthy volunteers to investigate the effects of coadministration of esaxerenone on the PK of digoxin, a typical substrate of $\mathrm{P}$-gp, because it has a narrow therapeutic window and is likely to be coadministered with esaxerenone. In that study, esaxerenone had no clinically relevant impact on the steady-state PK of digoxin (Kirigaya et al., 2020b), which was consistent with the static model analysis result. Regarding the metabolites of esaxerenone in the circulating blood, the abundance ratio of $O$-glucuronide was highest and accounted for $\sim 50 \%$ of the unchanged form (Yamada et al., 2019). Considering that enzyme inhibition of metabolites with higher polarity is usually weaker than that with the unchanged form ( $\mathrm{Yu}$ and Tweedie, 2013), the DDI risk by esaxerenone metabolites is low.

The TDI and induction of CYP3A by esaxerenone required modeling analyses to evaluate the DDI risk in clinical settings. Because it is difficult to accurately estimate the offset between inhibition and induction (i.e., the phenomenon in which decreased enzymatic activity due to inhibition is counteracted by increased enzyme expression due to induction), DDI guidance recommends separately evaluating inhibition and induction (Food and Drug Administration, 2020). Separate prediction of the effects of CYP3A TDI and induction using a mechanistic static model suggested DDI potential; therefore, a clinical DDI study with midazolam was conducted (Furuie et al., 2019). However, mechanistic static models tend to overestimate risks to avoid false negatives because of the unrealistic assumption that the maximum concentration of a perpetrator is sustained (Vieira et al., 2014), and PBPK modeling approaches simulating dynamic concentration change are used for more realistic predictions. Although PBPK modeling analysis of DDIs via CYP3A has been extensively performed, the predictive accuracy of weak DDIs via intestinal CYP3A were unclear because many focused on strong interactions. Therefore, we confirmed the predictability using 17 marketed drugs that exhibited weak inhibition or both inhibition and induction in our previous study and showed that GastroPlus favorably predicted the effects of weak CYP3A perpetrators on midazolam (Yamada et al., 2020). More than half of the 17 perpetrators were known to be substrates of CYP3A. However, the distribution and elimination were described by compartmental models using clinical PK data after oral administration, resulting in satisfactory prediction.

In the current study, the DDI between midazolam and esaxerenone via both the inhibition and induction of CYP3A was simulated by GastroPlus using similar methods as those used in our previous study above (Yamada et al., 2020). To compare the predictability of the models created with limited data, the three GastroPlus models for dynamic simulation were created step-by-step considering each actual drug 

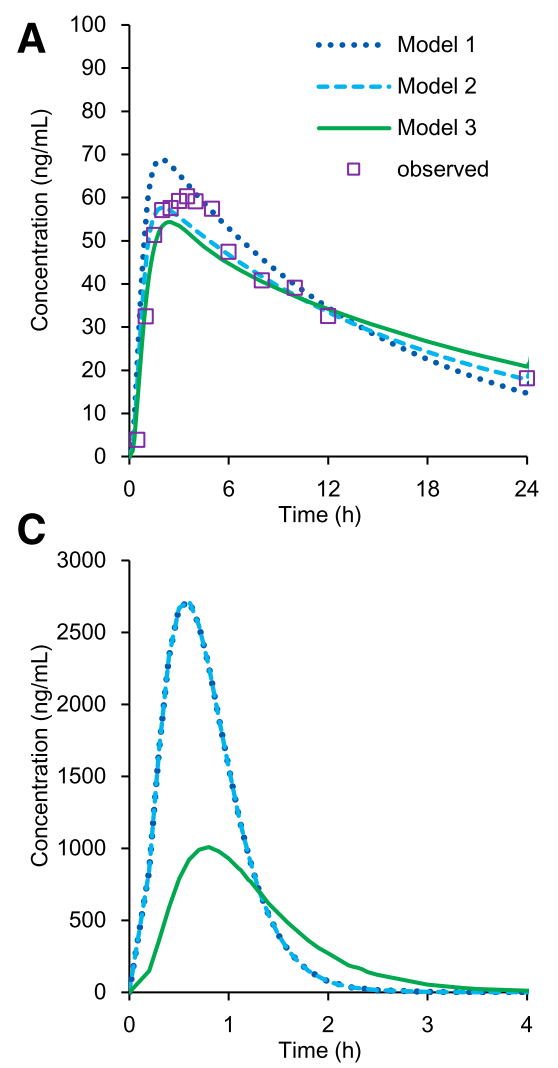
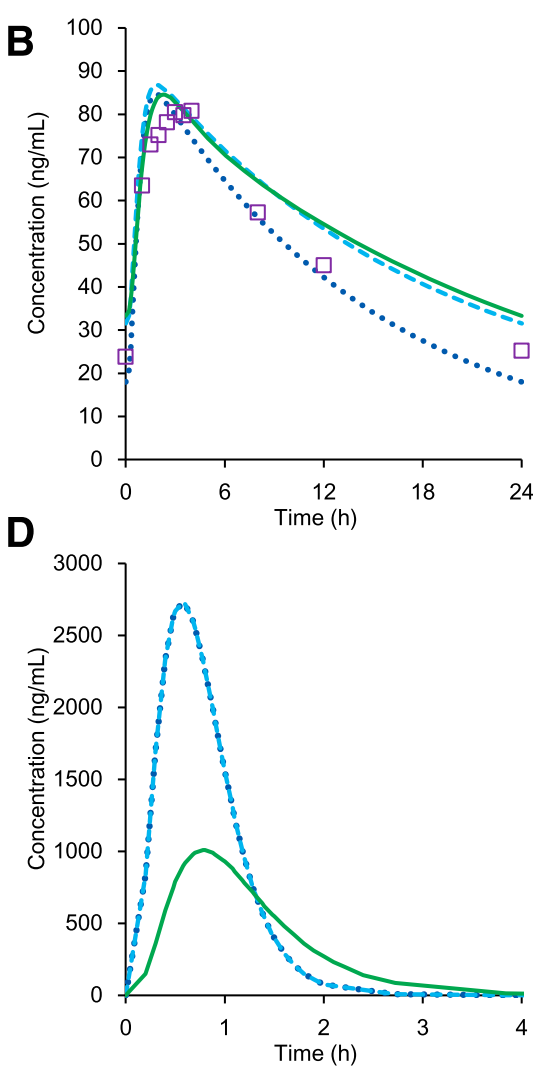

Fig. 3. Simulated concentrations of esaxerenone in plasma and enterocytes. Simulated concentrations of esaxerenone in plasma after single (A) and 14-day (B) administration as well as enterocytes (jejunum compartment 1 in GastroPlus is shown as an example) after single (C) and 14-day (D) administration. The enterocyte concentrations in models 1 and 2 are the same. Observed data are from clinical studies (Kato et al., 2018). The steady-state concentrations after multiple oral administrations at $5 \mathrm{mg}$ were calculated from the values after administration of $10 \mathrm{mg}$ for 10 days assuming linear PK. development process. Human PK parameters predicted from nonclinical data are generally used to construct a model before the first-in-human study; after a trial starts, the model is refined using human PK data. In the preclinical stage, we used several approaches to predict human PK; as an example, single-species allometric scaling using monkey PK data after intravenous administration (Lombardo et al., 2013a,b) was chosen for model 1 used in the present study. In many cases, only oral administration data are available for oral drugs in the early stages of clinical development; thus, the concentration-time profile after oral administration of esaxerenone (Kato et al., 2018) was used for model 2. Intestinal DDIs were able to be predicted even if there is a discrepancy between estimated and true PK parameters (i.e., CL, volume of distribution, $\mathrm{K}_{12}$, and $\mathrm{K}_{21}$ ). In other words, when PK parameters are optimized for the PK profile after oral administration (as in model 2),

TABLE 5

Simulated AUCRs of midazolam with three different models of esaxerenone by GastroPlus

Model 1, PK parameters predicted from monkey data; model 2, PK parameters optimized using the data after oral administration; model 3 , PK parameters calculated using the data after intravenous administration.

\begin{tabular}{|c|c|c|c|c|}
\hline & Mechanism & $\mathrm{AUCR}_{\mathrm{g}}$ & $\mathrm{AUCR}_{\mathrm{h}}$ & AUCR \\
\hline \multirow[t]{3}{*}{ Model 1} & Inhibitiona & 1.49 & 1.01 & 1.51 \\
\hline & Induction & 0.68 & 0.99 & 0.67 \\
\hline & Total & 1.22 & 1.01 & 1.23 \\
\hline \multirow[t]{3}{*}{ Model 2} & Inhibition $^{a}$ & 1.49 & 1.02 & 1.51 \\
\hline & Induction & 0.68 & 0.99 & 0.67 \\
\hline & Total & 1.22 & 1.01 & 1.23 \\
\hline \multirow[t]{3}{*}{ Model 3} & Inhibition $^{a}$ & 1.34 & 1.02 & 1.36 \\
\hline & Induction & 0.79 & 0.99 & 0.79 \\
\hline & Total & 1.16 & 1.01 & 1.17 \\
\hline
\end{tabular}

${ }^{a}$ Both reversible inhibition and TDI. The dosing conditions of the analysis were the same as those in the clinical study (i.e., esaxerenone $5 \mathrm{mg}$ for 14 days). good fitting is insufficient to justify the model for intestinal DDI prediction, and it is important to use reasonable solubility, membrane permeability, and if necessary, intestinal metabolism to simulate $\mathrm{C}_{\mathrm{ent}}$. Model 3 showed a decrease of $\mathrm{C}_{\text {ent }}$ (Fig. 3, C and D) due to the FPE and a slower dissolution rate, which results in a smaller $\mathrm{AUCR}_{\mathrm{inh}}$. However, the $\mathrm{AUCR}_{\text {ind }}$ was predicted to be larger, and the $\mathrm{AUCR}_{\text {tot }}$ values after the cancellation of inhibition by induction were comparable to those of models 1 and 2 . Although the use of intravenous data are generally considered to improve prediction accuracy, no clear difference was observed in the current study. From these results, for compounds with both weak inhibition and induction, such as esaxerenone, reasonable models can be created using only data after oral administration. In fact, in our previous study of 17 marketed drugs, good predictability in perpetrator DDI simulations was obtained by the models created using data after oral administration (Yamada et al., 2020).

When both inhibition and induction occur, the resultant effects can vary depending on the administration duration. For example, ritonavir has potential for reversible inhibition, TDI, and induction of CYP3A4. When ritonavir is orally administered for 9 days and midazolam is orally administered on days 1, 2, 3, 5, 8, and 9, the AUCR of midazolam peaks on day 3 and declines significantly thereafter (Katzenmaier et al., 2011). Similarly, in another study, the AUCR of midazolam after a single dose of tipranavir/ritonavir was larger than that after multiple doses of tipranavir/ritonavir for 10 days (Dumond et al., 2010). These results suggest that the cancellation of inhibition by induction occurs in clinical settings and that clinical DDI studies should be designed not to miss the maximum effect. To understand the mechanisms of complex DDIs and appropriately design clinical studies, PBPK models have been used (Guest et al., 2011; Gertz et al., 2013; Liu et al., 2017). The current study simulated DDIs with different dosing periods of esaxerenone and timing of midazolam administration, and the change in the AUCR of midazolam was small. This is because the reversible inhibition of 


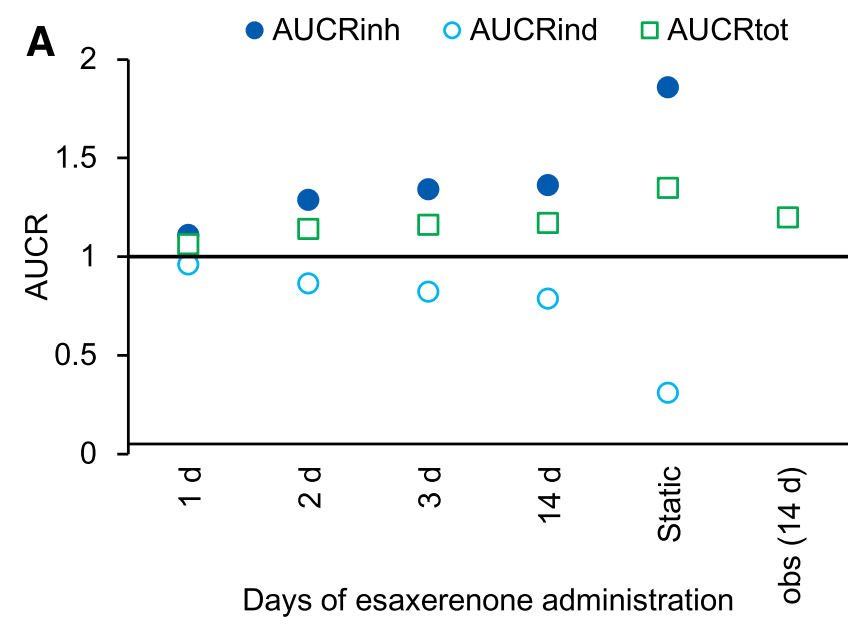

B
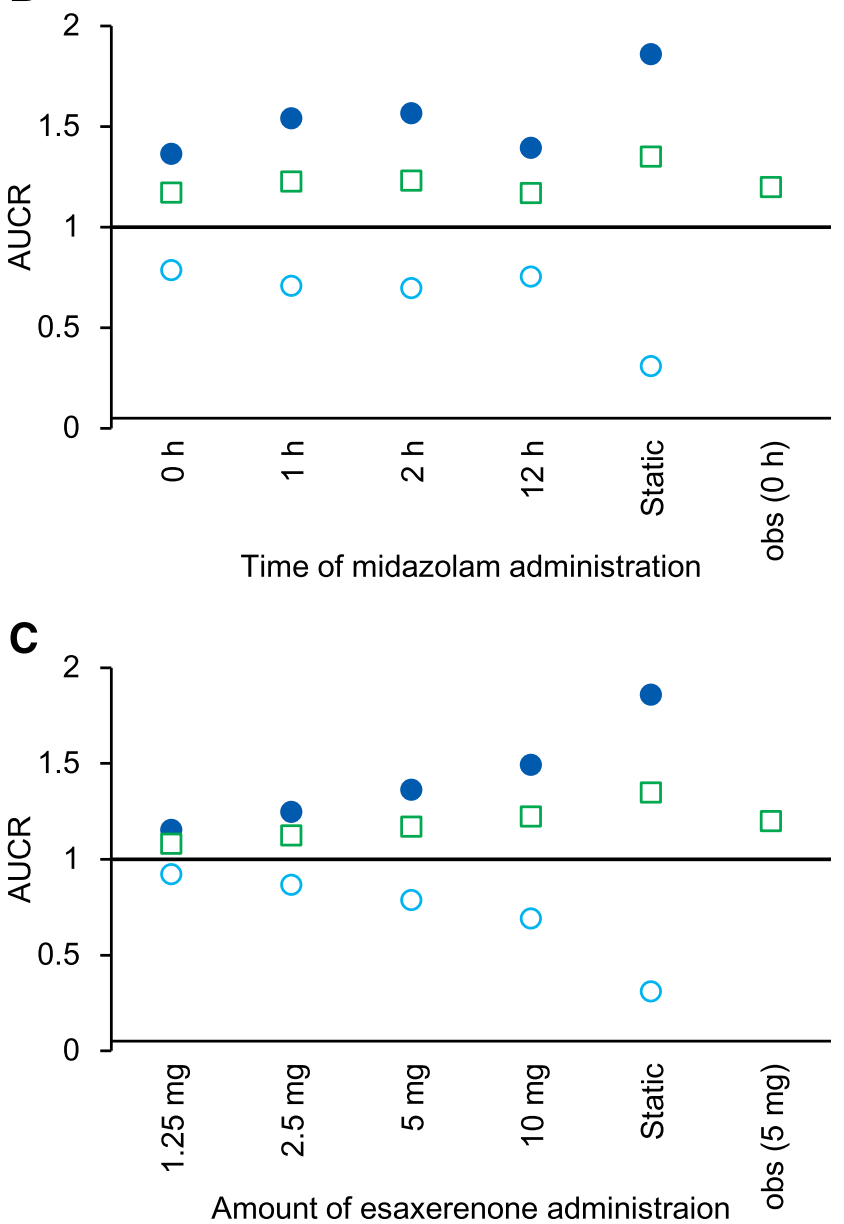

Fig. 4. Simulated midazolam AUCRs when esaxerenone was coadministered with different durations of esaxerenone administration (A), midazolam administration timing after esaxerenone administration (B), and esaxerenone dose levels (C). DDIs of midazolam and esaxerenone were simulated using the PBPK model with inhibition only $\left(\mathrm{AUCR}_{\mathrm{inh}}\right)$, induction only $\left(\mathrm{AUCR}_{\mathrm{ind}}\right)$, or both inhibition and induction $\left(\mathrm{AUCR}_{\mathrm{tot}}\right)$. The observed AUCR from the clinical study and the AUCRs calculated using the static model are also plotted. "Static" indicates the results of static model analysis, and "obs" indicates the clinically observed AUCR (Furuie et al., 2019).

esaxerenone is weak, and only TDI and induction could affect DDI in clinical settings. When a simulation was conducted using a virtual perpetrator with only reversible inhibition and induction stronger than esaxerenone (i.e., $\mathrm{K}_{\mathrm{i}}$ and $\mathrm{EC}_{50}$ 1/50th of those of esaxerenone), a significant change in $\mathrm{AUCR}_{\text {tot }}$ was predicted depending on the duration and timing of administration (Supplemental Fig. 1). The enzyme activity changes in the cases of reversible inhibition, TDI, and induction and their offset is shown in Supplemental Fig. 7. The change due to reversible inhibition decreases and the change due to TDI slightly increases by delaying the administration timing of a substrate. In either case, if intestinal CYP3A is maximally inhibited, a clear change might not be seen. The induction is strengthened by delaying the administration timing so that when the weak TDI and weak induction are balanced, the total change will be mitigated. There are few published results of clinical studies conducted under different dosing conditions, and the reliability of predicting the cancellation of strong inhibition and strong induction requires further investigation. In the current study, the midazolam area under the curve was less affected by coadministered esaxerenone dose because of the cancellation of inhibition and induction (Fig. 4C), suggesting that esaxerenone has a low DDI risk even if $10 \mathrm{mg}$ (twice the maximum approved dose) is administered. Considering that esaxerenone is a drug for hypertension, it is likely that it would be coadministered with statins and other drugs with lower $F_{g}$ than midazolam. Therefore, simulations were performed to address potential DDI risk in those scenarios using virtual substrates (Supplemental Fig. 8). Compounds that maximally inhibit intestinal CYP3A are expected to cause larger AUCR of substrates with high intestinal first pass (i.e., low $F_{g}$ ). However, because esaxerenone does not completely inhibit intestinal CYP3A, the DDI risk with these types of victim drugs is expected to be low, and the offset by induction would further mitigate the interaction risk. Unfortunately, because there are few examples of clinical DDI studies using a weak perpetrator with multiple CYP3A substrates, this hypothesis needs to be tested in the future.

In conclusion, the DDI potential of esaxerenone as a perpetrator is low. Although esaxerenone exhibits reversible inhibitory effects on several enzymes and transporters in vitro, they are unlikely to affect the kinetics of other drugs considering clinical exposure. Because of the cancellation of TDI and induction, DDIs between esaxerenone and CYP3A substrates are considered to be only weakly affected by dosing conditions such as duration and dose staggering. The utility of GastroPlus for predicting CYP3A-mediated DDIs mainly occurring in the intestine was also confirmed. The results suggest that GastroPlus can be used from early drug development stages to predict weak DDIs in the intestine, as the model created using animal data showed favorable predictability. For predictions considering the time-dependent change of enzyme activity in the intestine, simulations using a PBPK model that can describe the concentration-time profile are essential.

\section{Acknowledgments}

The authors thank Sekisui Medical Co., Ltd., Shin Nippon Biomedical Laboratories, Ltd., LSI Medience Corporation, and Daiichi Sankyo RD Novare Co., Ltd., for their expert experimental support.

\section{Authorship Contributions}

Participated in research design: Yamada, Ishizuka, Inoue.

Conducted experiments: Yamada, Rozehnal, Fischer.

Performed data analysis: Yamada, Ishizuka, Rozehnal, Fischer.

Wrote or contributed to the writing of the manuscript: Yamada, Ishizuka, Inoue, Rozehnal, Fischer, Sugiyama.

\section{References}

Arai K, Homma T, Morikawa Y, Ubukata N, Tsuruoka H, Aoki K, Ishikawa H, Mizuno M, and Sada T (2015a) Pharmacological profile of CS-3150, a novel, highly potent and selective non-steroidal mineralocorticoid receptor antagonist. Eur J Pharmacol 761:226-234.

Arai K, Morikawa Y, Ubukata N, Tsuruoka H, and Homma T (2016) CS-3150, a novel nonsteroida mineralocorticoid receptor antagonist, shows preventive and therapeutic effects on renal injury in deoxycorticosterone acetate/salt-induced hypertensive rats. J Pharmacol Exp Ther 358:548-557. 
Arai K, Tsuruoka H, and Homma T (2015b) CS-3150, a novel non-steroidal mineralocorticoid receptor antagonist, prevents hypertension and cardiorenal injury in Dahl salt-sensitive hypertensive rats. Eur J Pharmacol 769:266-273.

Austin RP, Barton P, Mohmed S, and Riley RJ (2005) The binding of drugs to hepatocytes and its relationship to physicochemical properties. Drug Metab Dispos 33:419-425.

Brown HS, Ito K, Galetin A, and Houston JB (2005) Prediction of in vivo drug-drug interactions from in vitro data: impact of incorporating parallel pathways of drug elimination and inhibitor absorption rate constant. Br J Clin Pharmacol 60:508-518.

Dumond JB, Vourvahis M, Rezk NL, Patterson KB, Tien HC, White N, Jennings SH, Choi SO, Li J, Wagner MJ, et al. (2010) A phenotype-genotype approach to predicting CYP450 and P-glycoprotein drug interactions with the mixed inhibitor/inducer tipranavir/ritonavir. Clin Pharmacol Ther 87:735-742.

Food and Drug Administration (2020) In vitro drug interaction studies - cytochrome P450 enzyme- and transporter-mediated drug interactions guidance for industry, US Department of Health and Human Services, Center for Drug Evaluation and Research, Food and Drug Administration, Silver Spring, MD.

Furuie H, Toyama K, Okuda Y, Kuroda K, Shimizu T, Kato M, and Ishizuka H (2019) The effect of multiple oral administration of esaxerenone on the pharmacokinetics of midazolam in healthy Japanese males (Abstract). Eur J Clin Pharmacol 75 (Suppl 1):S38.

Gertz M, Cartwright CM, Hobbs MJ, Kenworthy KE, Rowland M, Houston JB, and Galetin A (2013) Cyclosporine inhibition of hepatic and intestinal CYP3A4, uptake and efflux transporters: application of PBPK modeling in the assessment of drug-drug interaction potential. Pharm Res 30:761-780.

Grimstein M, Yang Y, Zhang X, Grillo J, Huang SM, Zineh I, and Wang Y (2019) Physiologically based pharmacokinetic modeling in regulatory science: an update from the U.S. Food and Drug Administration's Office of Clinical Pharmacology. J Pharm Sci 108:21-25.

Guest EJ, Rowland-Yeo K, Rostami-Hodjegan A, Tucker GT, Houston JB, and Galetin A (2011) Assessment of algorithms for predicting drug-drug interactions via inhibition mechanisms: comparison of dynamic and static models. Br J Clin Pharmacol 71:72-87.

Guo Y, Chu X, Parrott NJ, Brouwer KLR, Hsu V, Nagar S, Matsson P, Sharma P, Snoeys J, Sugiyama Y, et al.; International Transporter Consortium (2018) Advancing predictions of tissue and intracellular drug concentrations using in vitro, imaging and physiologically based pharmacokinetic modeling approaches. Clin Pharmacol Ther 104:865-889.

Hsueh CH, Hsu V, Pan Y, and Zhao P (2018) Predictive performance of physiologically-based pharmacokinetic models in predicting drug-drug interactions involving enzyme modulation. Clin Pharmacokinet 57:1337-1346.

Ito K, Iwatsubo T, Kanamitsu S, Ueda K, Suzuki H, and Sugiyama Y (1998) Prediction of pharmacokinetic alterations caused by drug-drug interactions: metabolic interaction in the liver. Pharmacol Rev 50:387-412.

Ito S, Itoh H, Rakugi H, Okuda Y, and Yamakawa S (2019a) Efficacy and safety of esaxerenone (CS-3150) for the treatment of essential hypertension: a phase 2 randomized, placebo-controlled, double-blind study. J Hum Hypertens 33:542-551.

Ito S, Itoh H, Rakugi H, Okuda Y, Yoshimura M, and Yamakawa S (2020) Double-blind randomized phase 3 study comparing esaxerenone (CS-3150) and eplerenone in patients with essential hypertension (ESAX-HTN study). Hypertension 75:51-58

Ito S, Shikata K, Nangaku M, Okuda Y, and Sawanobori T (2019b) Efficacy and safety of esaxerenone (CS-3150) for the treatment of type 2 diabetes with microalbuminuria: a randomized, double-blind, placebo-controlled, phase II trial. Clin J Am Soc Nephrol 14 $1161-1172$.

Jones HM, Chen Y, Gibson C, Heimbach T, Parrott N, Peters SA, Snoeys J, Upreti VV, Zheng M, and Hall SD (2015) Physiologically based pharmacokinetic modeling in drug discovery and development: a pharmaceutical industry perspective. Clin Pharmacol Ther 97:247-262.

Kato M, Furuie H, Shimizu T, Miyazaki A, Kobayashi F, and Ishizuka H (2018) Single- and multiple-dose escalation study to assess pharmacokinetics, pharmacodynamics and safety of ora esaxerenone in healthy Japanese subjects. Br J Clin Pharmacol 84:1821-1829.

Katzenmaier S, Markert C, Riedel KD, Burhenne J, Haefeli WE, and Mikus G (2011) Determining the time course of CYP3A inhibition by potent reversible and irreversible CYP3A inhibitors using A limited sampling strategy. Clin Pharmacol Ther 90:666-673.

Kirigaya Y, Shiramoto M, Ishizuka T, Uchimaru H, Irie S, Kato M, Shimizu T, Nakatsu T, Nishikawa Y, and Ishizuka H (2020a) Effects of itraconazole and rifampicin on the singledose pharmacokinetics of the nonsteroidal mineralocorticoid receptor blocker esaxerenone in healthy Japanese subjects. Br J Clin Pharmacol DOI: 10.1111/bcp.14302 [published ahead of print].

Kirigaya Y, Shiramoto M, Ishizuka T, Uchimaru H, Irie S, Kato M, Shimizu T, Nakatsu T, Nishikawa $\mathrm{Y}$, and Ishizuka H (2020b) Pharmacokinetic interactions of esaxerenone with amlodipine and digoxin in healthy Japanese subjects. BMC Pharmacol Toxicol in press.
Kurata A, Furuie H, Ishizuka T, Nakatsu T, Shimizu T, Kato M, Nishikawa Y, and Ishizuka H (2019) Absolute bioavailability of esaxerenone and food effects on its pharmacokinetics after a single oral dose in healthy Japanese subjects: an open-label crossover study. Adv Ther 36: $1618-1627$.

Liu B, Crewe HK, Ozdemir M, Rowland Yeo K, Tucker G, and Rostami-Hodjegan A (2017) The absorption kinetics of ketoconazole plays a major role in explaining the reported variability in the level of interaction with midazolam: interplay between formulation and inhibition of gut wall and liver metabolism. Biopharm Drug Dispos 38:260-270.

Lombardo F, Waters NJ, Argikar UA, Dennehy MK, Zhan J, Gunduz M, Harriman SP, Berellini G, Liric Rajlic I, and Obach RS (2013a) Comprehensive assessment of human pharmacokinetic prediction based on in vivo animal pharmacokinetic data, part 2: clearance. J Clin Pharmacol 53: $178-191$.

Lombardo F, Waters NJ, Argikar UA, Dennehy MK, Zhan J, Gunduz M, Harriman SP, Berellini G, Rajlic IL, and Obach RS (2013b) Comprehensive assessment of human pharmacokinetic prediction based on in vivo animal pharmacokinetic data, part 1: volume of distribution at steady state. J Clin Pharmacol 53:167-177.

Pharmaceutical Evaluation Division, Pharmaceuticals Safety and Environmental Health Bureau Ministry of Labor and Welfare (2018) Guideline on drug interaction for drug development and appropriate provision of information, Pharmaceutical Evaluation Division, Pharmaceuticals Safety and Environmental Health Bureau, Ministry of Labor and Welfare, Tokyo, Japan.

Rakugi H, Ito S, Itoh H, Okuda Y, and Yamakawa S (2019) Long-term phase 3 study of esaxerenone as mono or combination therapy with other antihypertensive drugs in patients with essential hypertension. Hypertens Res 42:1932-1941.

Rostami-Hodjegan A and Tucker G (2004) 'In silico' simulations to assess the 'in vivo' consequences of 'in vitro' metabolic drug-drug interactions. Drug Discov Today Technol 1:441-448.

Shebley M, Sandhu P, Emami Riedmaier A, Jamei M, Narayanan R, Patel A, Peters SA, Reddy VP, Zheng M, de Zwart L, et al. (2018) Physiologically based pharmacokinetic model qualification and reporting procedures for regulatory submissions: a consortium perspective. Clin Pharmacol Ther 104:88-110.

Thummel KE, O'Shea D, Paine MF, Shen DD, Kunze KL, Perkins JD, and Wilkinson GR (1996) Oral first-pass elimination of midazolam involves both gastrointestinal and hepatic CYP3Amediated metabolism. Clin Pharmacol Ther 59:491-502.

Vieira ML, Kirby B, Ragueneau-Majlessi I, Galetin A, Chien JY, Einolf HJ, Fahmi OA, Fischer V, Fretland A, Grime K, et al. (2014) Evaluation of various static in vitro-in vivo extrapolation models for risk assessment of the CYP3A inhibition potential of an investigational drug. Clin Pharmacol Ther 95:189-198.

Yamada M, Inoue SI, Sugiyama D, Nishiya Y, Ishizuka T, Watanabe A, Watanabe K, Yamashita S, and Watanabe N (2020) Critical impact of drug-drug interactions via intestinal CYP3A in the risk assessment of weak perpetrators using physiologically based pharmacokinetic models. Drug Metab Dispos 48:288-296.

Yamada M, Mendell J, Takakusa H, Shimizu T, and Ando O (2019) Pharmacokinetics, metabolism, and excretion of [14C]esaxerenone, a novel mineralocorticoid receptor blocker in humans. Drug Metab Dispos 47:340-349.

Yamada M, Takei M, Suzuki E, Takakusa H, Kotsuma M, Washio T, Murayama N, Inoue SI, and Izumi T (2017) Pharmacokinetics, distribution, and disposition of esaxerenone, a novel, highly potent and selective non-steroidal mineralocorticoid receptor antagonist, in rats and monkeys. Xenobiotica 47:1090-1103.

Yang J, Jamei M, Yeo KR, Rostami-Hodjegan A, and Tucker GT (2007b) Misuse of the wellstirred model of hepatic drug clearance. Drug Metab Dispos 35:501-502.

Yang J, Jamei M, Yeo KR, Tucker GT, and Rostami-Hodjegan A (2007a) Prediction of intestinal first-pass drug metabolism. Curr Drug Metab 8:676-684.

Yu H and Tweedie D (2013) A perspective on the contribution of metabolites to drug-drug interaction potential: the need to consider both circulating levels and inhibition potency. Drug Metab Dispos 41:536-540.

Zhang X, Quinney SK, Gorski JC, Jones DR, and Hall SD (2009) Semiphysiologically based pharmacokinetic models for the inhibition of midazolam clearance by diltiazem and its major metabolite. Drug Metab Dispos 37:1587-1597.

Address correspondence to: Makiko Yamada, Drug Metabolism and Pharmacokinetics Research Laboratories, Daiichi Sankyo Co., Ltd., 1-2-58, Hiromachi, Shinagawa-ku, Tokyo 140-8710, Japan. E-mail: yamada.makiko.jr@daiichisankyo. co.jp 